\title{
IMPROBABILITY OF COLLISIONS IN NEWTONIAN GRAVITATIONAL SYSTEMS
}

\author{
BY \\ DONALD GENE SAARI $\left({ }^{1}\right)$
}

\begin{abstract}
It is shown that the set of initial conditions leading to a collision in finite time has measure zero.
\end{abstract}

1. It is well known that binary collisions of point masses in a Newtonian gravitational system are improbable in the sense that the set of initial conditions leading to this catastrophe at some finite time has (Lebesgue volume) measure zero. One would expect the same to be true for multiple collisions if only for some sort of aesthetic reasoning-there seems to be a binary collision contained within a multiple collision. What is shown here is that this is indeed the case; that is, the set of initial conditions leading to collision in finite time has measure zero. This problem has gained attention in recent years with its inclusion in J. E. Littlewood's list of problems [2, Problem 13].

It must be emphasized that the fact that the force law is the inverse square force law plays a crucial role in the proof of this result. To see that this is not true for all force laws, let $2 I=\sum m_{i} r_{i}^{2}$, where $m_{i}$ is the mass and $r_{i}$ the position vector of the $i$ th mass relative to the center of mass of the systems. It is well known (see [3], [6], [8], for example) that in the inverse $p$ force law $I=(3-p) T+(p-1) h$. Here $T$ is the kinetic energy, $h$ is the total energy of the system and the dots denote differentiation with respect to time. Note that if $p \geqq 3, I \cong(p-1) h$. Integration yields $I \leqq(p-1) h t^{2} / 2+I(0) t+I(0)$. It follows that all initial conditions possessing negative $h$ have the property that in finite time $I \rightarrow 0$. Hence if the solution lasts long enough then the system will suffer a complete collapse. In particular, if $n=2$ and $h<0$, there will be a collision. But the set of initial conditions yielding negative $h$ has measure greater than zero. (Of course in the inverse square law the above is not true as for $n=2$, negative energy leads in general to elliptic motion.)

The reason that binary collisions are improbable in the inverse square law is that the system retains its analytic dependence on initial conditions at collision. (This comes from the fact that a binary collision can be regularized. See, for

Received by the editors September 4, 1970 and, in revised form, January 19, 1971.

AMS 1969 subject classifications. Primary 7034; Secondary 3440, 8500.

Key words and phrases. $n$-body problem, Newtonian gravitational systems, measure preserving, collisions.

(1) This was written while the author was a visitor at Seminar für angewandte Mathematik, ETH, Zürich, Switzerland. This research was partially supported under NSF GP 19526. 
example, the work of Sundman [7].) Hence the $(6 n-2)$-dimensional manifold $\boldsymbol{r}_{1}=\boldsymbol{r}_{2}$ in $R^{6 n+1}$ corresponds to binary collisions of $m_{1}$ and $m_{2}$ at any time. Here $R^{6 n+1}=R^{6 n} \times\{t\}$; that is, phase space augmented by the time coordinate. The analytic dependence on initial conditions can be used to map this manifold into the set of initial conditions, $R^{6 n} \times\{0\}$, forming a submanifold of dimension $6 n-2$, which is of (volume) measure zero. As there are only a finite number of combinations of indices for binary collisions, the result holds. (This result seems to be due to G. D. Birkhoff [1, pp. 270-272].) The problem with multiple collisions is that the analytic dependence may not hold.

The idea employed here for multiple collisions is a slight variation of the above and is essentially the following: Let $\mathfrak{M}$ denote the set of initial conditions leading to a common collision of particles $m_{1}-m_{k}$ at some time $t_{0}$. Prior to collision, the flow $\phi_{t}(\mathfrak{M})$ is measure preserving. From the analytic theory of collisions we then show that as $t_{i} \rightarrow t_{0}, \phi_{t}(\mathfrak{M}) \subset A_{i}$ where meas $A_{i} \rightarrow 0$ as $i \rightarrow \infty$. That is meas $\mathfrak{M}$ $=$ meas $\phi_{t}(\mathfrak{M})=0$.

The result described above holds for all collisions of the n-body problem but not necessarily for all singularities of the n-body problem. The question of whether a singularity is necessarily a collision is still an open question [4], [5], [9].

Further notation and necessary analytic results from the theory of collisions will be given in $\$ 2$. The statement and proof of the theorem will be given in $\$ 3$. An extension of the result for other force laws will be discussed in $\$ 4$. Finally I would like to thank Harry Pollard, Carl Simon and Sheldon Newhouse for some interesting discussions on this topic.

2. Notation. Our basic assumptions are that $n$, the number of particles, is finite and that we have an inertial coordinate system. Let $m_{k}, \boldsymbol{r}_{k}$ and $\boldsymbol{v}_{k}$ denote respectively the mass, position and velocity of the $k$ th particle. We define further $r_{k}=\left|\boldsymbol{r}_{k}\right|, v_{k}=\left|\boldsymbol{v}_{k}\right|=\left|\dot{\boldsymbol{r}}_{k}\right|$ and $2 T=\sum m_{k} v_{k}^{2}$.

Following Pollard and Saari [4], we say that we have a collision at time $t_{0}$ if as $t \rightarrow t_{0}$ each particle approaches a definite position in the inertial coordinate frame with at least two particles sharing the same limit point.

The analytic result upon which we will rely most heavily is quoted below and can be found in [4].

THEOREM. If there is a collision at $t=t_{0}$ then $J=\frac{1}{2} \sum m_{i}\left(\boldsymbol{r}_{i}-L_{i}\right)^{2} \sim A\left|t-t_{0}\right|^{4 / 3}$ and $T \sim \frac{4}{9} A\left|t-t_{0}\right|^{-2 / 3}$. $A$ is some positive constant and $\boldsymbol{r}_{i} \rightarrow L_{i}$ as $t \rightarrow t_{0}$.

\section{Statement and proof of the theorem.}

THEOREM. The set of initial conditions leading to collision in finite time has (Lebesgue volume) measure zero.

Actually what will be proved is that the set of initial conditions leading to the simultaneous collision of $m_{1}, \ldots, m_{k}(k \leqq n)$ at a common point has measure zero. 
With only minor modifications the proof carries over to the case where we have a simultaneous collision at more than one collision point. As there are only a finite number of combinations ( $n$ is finite) the theorem follows.

As we will be assuming that $m_{1}, \ldots, m_{k}$ collide, we will denote the subspace $\left(\boldsymbol{r}_{1}, \ldots, \boldsymbol{r}_{k}, \mathbf{0}, \ldots, 0, v_{1}, \ldots, v_{k}, 0, \ldots, 0\right)$ by $R^{6 k}$ and its orthogonal complement by $R^{6(n-k)}$.

The concepts employed in the proof are straightforward with the major complications coming from the facts that (1) the collision may occur at any finite time, (2) the collision may take place at any point along the line $\boldsymbol{r}_{1}=\cdots=\boldsymbol{r}_{k}$ in configuration space of $R^{6 k}$, (3) the constant $A$ and the $o\left(t^{4 / 3}\right)$ term in $J=A t^{4 / 3}+o\left(t^{4 / 3}\right)$ may depend upon the initial conditions, and (4) the state of the noncolliding particles is arbitrarily located in $R^{6(n-k)}$.

Proof. We consider the case where only particles $m_{1}, \ldots, m_{k}$ collide with a common limit point at some finite time. We further limit ourselves to the cases where the common collision point is on an arbitrary but fixed unit interval on the line $\boldsymbol{r}_{1}=\cdots=\boldsymbol{r}_{k}$. Let $B$ be an arbitrary positive constant and $S(p, 1)$ be a sphere in $R^{6(n-k)}$ of radius 1 about the rational point $p$. Motivated by the analytic behavior of collisions, we define for positive integer $\alpha, B^{\alpha}\left(t_{1}\right)$ to be the set of points in $R^{6 n}$ with the following properties:

1. Assuming the point to be an initial condition at time $t_{1}$, the solution exists in the interval $\left[0, t_{1}\right]$.

2. At $t_{1}$, the coordinates for $m_{k+1}, \ldots, m_{n}$ are in $S(p, 1)$.

3. At time $t_{1}$, the coordinates in $R^{6 k}$ satisfy

$$
\sum m_{i}\left(r_{i}-L\right)^{2} \leqq M B^{2}\left(2^{-\alpha}\right)^{4 / 3} \text { and } \sum m_{i} v_{i}^{2} \leqq \frac{2}{9} M B^{2}\left(2^{-1.1 \alpha}\right)^{-2 / 3}
$$

where $\boldsymbol{L}$ is any point on our arbitrary but fixed interval on $\boldsymbol{r}_{1}=\cdots=\boldsymbol{r}_{k}$ and $M$ $=\sum m_{i}$.

By construction $B^{\alpha}\left(t_{1}\right)$ is a measurable set. That (2) and (3) define a measurable set is obvious. A standard argument employing "continuity with respect to initial conditions" shows that (1) defines a measurable set. Hence $B^{\alpha}\left(t_{1}\right)$ is measurable.

We now estimate the measure of $B^{\alpha}\left(t_{1}\right)$. From condition (3),

$$
\left|\boldsymbol{r}_{i}-\boldsymbol{L}\right| \leqq B(2)^{-2 \alpha / 3} \text { and }\left|\boldsymbol{v}_{i}\right| \leqq\left(\frac{2}{9}\right)^{1 / 2} B(2)^{1.1 \alpha / 3} \text {. }
$$

Taking into account that the above estimates are for vectors and the length of our unit interval is unity, it follows that

$$
\text { meas } B^{\alpha}\left(t_{1}\right) \leqq D\left(2^{-\alpha}\right)^{0.9 k-2 / 3}
$$

where $D=(\operatorname{vol} S(p, 1)) B^{6 k-1}\left(\frac{2}{9}\right)^{3 k / 2}[1+2 B](2)^{6 k-1}$.

We now consider an arbitrary unit interval of time with rational endpoints, say $[1,2]$, and divide it into $2^{\alpha+4}$ equal parts. This defines points $t_{6}$ where $t_{0}=1$ and $t_{2^{\alpha+4}}=2$. Let $B^{\alpha}[1,2]$ be the set of initial conditions leading to $\bigcup B^{\alpha}\left(t_{i}\right)$. 
As the $n$-body problem is a conservative dynamical system, its flow is measure preserving. By condition 1 in the definition of $B^{\alpha}\left(t_{1}\right), B^{\alpha}\left(t_{i}\right)$ can be pulled back to the zero with the resulting set having the same measure. Hence $B^{\alpha}[1,2]$ is measurable (finite sum of measurable sets) and

$$
\text { meas } B^{\alpha}[1,2] \leqq 2^{5} D\left(2^{-\alpha}\right)^{0.9 k-5 / 3} \text {. }
$$

Define $\beta[1,2]=\lim \sup B^{\alpha}[1,2]$ as $\alpha \rightarrow \infty$. The measure of $\beta[1,2]$ is zero. This follows from the fact that the minimum value for the integer $k$ is 2 . Hence the exponent on the $\left(2^{-\alpha}\right)$ term is positive. Now, if one sums the right-hand side of (2) for $\alpha=1,2, \ldots$, one obtains a convergent series. By construction meas $\beta[1,2]$ $\leqq \sum_{\alpha=N}^{\infty}$ meas $B^{\alpha}[1,2]$, which can be made arbitrarily small for large enough $N$.

We now relate the above construction of $\beta[1,2]$ to the collision problem. Let $c$ be the set of initial conditions such that

1. Particles $m_{1}, \ldots, m_{k}$ collide at a common collision point which is located on our specified interval on $\boldsymbol{r}_{1}=\cdots=\boldsymbol{r}_{k}$.

2. The first collision occurs at time $t^{*}$ where $t^{*}$ is in the interval $\left[\frac{5}{4}, \frac{7}{4}\right]$.

3. The state of the noncolliding particles at collision is in $S\left(p, \frac{1}{2}\right)$.

4. $J \sim A\left|t-t^{*}\right|^{4 / 3}$ where $4 A \leqq B^{2} M$.

We claim that $\mathbb{C} \subset \beta[1,2]$. Consider a point in $\mathbb{E}$ and assume that its collision time is $t^{*}\left(\varepsilon\left[\frac{5}{4}, \frac{7}{4}\right]\right)$. By the constraint on $A$ and as $J \sim A\left|t-t^{*}\right|^{4 / 3}$ it follows that, for all $\alpha$ after some integer, if $\left|t-t^{*}\right|<2^{-\alpha}$ then $J \leqq M B^{2}\left(2^{-\alpha}\right)^{4 / 3}$. Likewise as $T \sim \frac{4}{9} A\left|t-t^{*}\right|^{-2 / 3}$ and as $\left|t-t^{*}\right|^{-2 / 3}|| t-\left.t^{*}\right|^{-(1.1) 2 / 3} \rightarrow 0$ as $t \rightarrow t^{*}$, for all $\alpha$ after some integer, $2^{-(\alpha+2)}<\left|t-t^{*}\right|<2^{-\alpha}$ implies that $T \leqq M B^{2} 2^{2.2 \alpha / 3}$. The position and velocity vectors of the noncolliding particles approach definite limits in $S\left(p, \frac{1}{2}\right)$ as $\mathrm{t} \rightarrow t^{*}$, hence for all $\alpha$ after some integer, $\left|t-t^{*}\right|<2^{-\alpha}$ implies that the state of the noncolliding particles is in $S(p, 1)$. (By the equations of motion, $\ddot{\boldsymbol{r}}_{i}=O(1)$ as $t \rightarrow t^{*}$ for $i>k$. Hence $\dot{r}$ approaches a limit as $t \rightarrow t^{*}$.)

The above implies that for all $\alpha$ greater than some integer, we can find a $t_{i}$ which lies between $t=0$ and $t^{*}$ such that at time $t_{i}$ the image of our point in $\mathbb{E}$ is in $B^{\alpha}\left(t_{i}\right)$. Note that there is more than one choice for $t_{i}$ and that it cannot be the partition point immediately preceding $t^{*}$. (Recall that the unit interval was subdivided into $2^{\alpha+4}$ equal parts.) This implies that for all $\alpha$ after some integer our point in $\mathbb{E}$ is also in $B^{\alpha}[1,2]$, hence it is in $\beta[1,2]$. This completes the proof of the claim.

Clearly meas $\mathfrak{E}=0$. Note that $\mathfrak{C}$ depends upon the time interval of collision (of length $\frac{1}{2}$ ), the state of the noncolliding particles (in $S\left(p, \frac{1}{2}\right)$ where $p$ is rational), the value of $B$ and the unit interval of collision points. By summing the various $c$ 's over all rational endpoints of intervals of time of length $\frac{1}{2}$, over all rational $p$ (for the state of the noncolliding particles), over all rational positive values of $B$ and over the denumerable number of unit intervals (containing the location of the collision points along $\boldsymbol{r}_{1}=\cdots=\boldsymbol{r}_{k}$ ), a new set, $\mathfrak{D}$, is obtained. As $\mathscr{D}$ is the countable union of sets of measure zero, meas $\mathfrak{D}=0$. By construction, $\mathfrak{D}$ contains all initial 
conditions leading to collision of $m_{1}, \ldots, m_{k}$ in finite time, hence the proof is completed.

4. Extension. The above proof and result is not peculiar to the inverse square force law. With only minor modifications it applies to other inverse $p$ force laws. To accomplish this, we need the analytic theory of collisions for other force laws [6].

A collision at $t=t_{0}$ in the inverse $p$ force law, $1<p<3$, is characterized by $J \sim A\left|t_{0}-t\right|^{4 /(p+1)}$ and $T \sim\left(4(3-p) /(p+1)^{2}\right) A\left|t_{0}-t\right|^{2((1-p) /(p+1))}$. $A$ is some positive constant.

With this result it follows that

COROLlaRY. In the inverse $p$ force law $1<p<15 / 7$, the set of initial conditions leading to collision in finite time has measure zero.

Proof. In equation (1), replace the value 1.1 with $q$. The present constraint on $q$ is that it is greater than unity. With this substitution, the measure of $B^{\alpha}[1,2]$ is bounded above by a positive constant times $2^{-\alpha}$ raised to the

$$
(p+1)^{-1}[-p(3 k q+1)+k(6+3 q)-3]
$$

power. To force $\beta[1,2]$ to have zero measure, this exponent must be positive. As the minimum value for $k$ is $2, p$ and $q$ can always be chosen to satisfy this constraint if $p<15 / 7$, and the proof is completed.

With more care, this range could possibly be extended to, say, $1<p<3$.

Of course the above results hold only for point masses. For homogeneous spherical bodies of positive radius the measure of initial conditions leading to collisions is positive. (Here we define a collision to be between mass $i$ of radius $a_{i}$ and mass $j$ of radius $a_{j}$ if $\left|\boldsymbol{r}_{i}-\boldsymbol{r}_{j}\right|=a_{i}+a_{j}$.

\section{REFERENCES}

1. G. D. Birkhoff, Dynamical systems, Amer. Math. Soc. Colloq. Publ., vol. 9, Amer. Math. Soc., Providence, R. I., 1927.

2. J. E. Littlewood, Some problems in real and complex analysis, Heath, Lexington, Mass., 1968. MR 39 \#5777.

3. H. Pollard, Mathematical introduction to celestial mechanics, Prentice-Hall, Englewood Cliffs, N. J., 1966. MR 33 \#5357.

4. H. Pollard and D. G. Saari, Singularities of the n-body problem. I, Arch Rational Mech. Anal. 30 (1968), 263-269. MR 37 \#7118.

5. - Singularities of the n-body problem. II, Inequalities, no. 2, Academic Press, New York, 1970.

6. D. G. Saari, Expanding gravitational systems, Trans. Amer. Math. Soc. 156 (1971), 219-240.

7. K. Sundman, Le problème des trois corps, Acta Soc. Sci. Fenn. 35 (1909), no. 9.

8. E. T. Whittaker, $A$ treatise on the analytical dynamics of particles and rigid bodies, 4 th ed., Dover, New York, 1944. MR 6, 74.

9. H. J. Sperling, On the real singularities of the n-body problem, J. Reine Angew. Math. 245 (1970), 15-40.

NORTHWESTERn UNIVERSITY, Evanston, IllinoIS 60201 